\title{
STABILITY OF PURE NILPOTENT STRUCTURES ON COLLAPSED MANIFOLDS
}

\author{
ZUOHAI JIANG AND SHICHENG XU
}

\begin{abstract}
The goal of this paper is to study the stability of pure nilpotent structures on a manifold associated to different collapsed metrics. We prove that if two metrics on a $n$-manifold of bounded sectional curvature are $L_{0}$-bi-Lipchitz equivalent and sufficient collapsed (depending on $L_{0}$ and $n$ ), then up to a diffeomorphism, the underlying nilpotent Killing structures coincide with each other or one is embedded into another as a subsheaf. It improves Cheeger-Fukaya-Gromov's locally compatibility of pure nilpotent Killing structures for one collapsed metric of bounded sectional curvature to two Lipschitz equivalent metrics. As an application, we prove that those pure nilpotent Killing structures constructed by various smoothing method to a Lipschitz equivalent metric of bounded sectional curvature are uniquely determined by the original metric modulo a diffeomorphism.
\end{abstract}

\section{INTRODUCTION}

0.1. Background. Let $\left(M^{n}, g\right)$ be a complete Riemannian $n$-manifold whose sectional curvature $|\sec (M, g)| \leq 1$. It is called $\epsilon$-collapsed, if for any $x \in M$ the injectivity radius satisfy inj. $\operatorname{rad}_{g}(x) \leq \epsilon$. Collapsed manifolds under bounded sectional curvature are extensively studied by Gromov, Cheeger-Gromov and Fukaya ([17], [12, 14], [8, 9], [7]). Since then many applications on manifolds of bounded sectional curvature were obtained (e.g., [11], [32], [33], etc. and survey papers [15], [35]).

A maximally collapsed manifold $\left(M^{n}, g\right)$, whose diameter and sectional curvature satisfy $\operatorname{diam}(M, g) \cdot|\sec (M, g)|^{1 / 2}<\epsilon(n)$, a constant depending on $n$, is characterized by Gromov's almost flat manifolds ([17,37]), such that $M$ is a infranilmanifold $\Gamma \backslash N$, where $N$ is a nilpotent Lie group and $\Gamma$ is a discrete affine transformation subgroup with a universal bounded index $[\Gamma: \Gamma \cap N]<w(n)$, a constant depending only on $n$. By a parametrized version of Gromov's almost-flat manifold, which is called Fukaya's fibration theorem $[12,14]$, the collapsing of a Riemannian manifold $(M, g)$ to a lower-dimensional manifold $(Y, h)$ corresponds to an affine fiber bundle $(M, Y, f)$ whose fiber is an almost flat manifold containing all collapsed directions. A fiber bundle $(M, Y, f)$ with fiber a infra-nilmanifold $\Gamma \backslash N$, is called affine if its structure group is contained in the affine transformation group of $\Gamma \backslash N$.

Date: August 7, 2018.

2010 Mathematics Subject Classification. 53C23, 53C21, 53C20, 5324. 
In general an $\epsilon$-collapsed manifold $(M, g)$ are characterized by a nilpotent Killing structure $\mathfrak{n}$, i.e., a sheaf of nilpotent Lie algebras of local vector fields pointing all $\epsilon$ collapsed directions, which are Killing fields with respect to a nearby metric $g_{\epsilon}$, and generate an action of a nilpotent Lie group on a normal cover of some neighborhood around points in $M$ (see [7] or section 2.2). A collapsed manifold $M$ decomposes along $\mathfrak{n}$ into "orbits", which are infra-nilmanifolds maybe of different dimension, tangent to the stalks of $\mathfrak{n}$ and absorbing all $\epsilon$-collapsed directions. The rank of $\mathfrak{n}$ is defined to be minimal dimension of its orbits. A nilpotent Killing structure $\mathfrak{n}$ is called pure, if the dimension of its stalk is locally constant. For a pure structure $\mathfrak{n}$, we define its dimension to be that of its stalk. Since collapsing can take place simultaneously on several length scales, nilpotent Killing structures on a fixed $\epsilon$ collapsed metric also depend on the choice of $\epsilon$, the scale that one inspects. An $F$-structure constructed in [9] is an local action of a sheaf of tori (complete on a finite norm cover) which corresponds to the smallest length scale of collapsing, i.e., the injectivity radius at each point.

The above theorems fails for manifolds of bounded Ricci curvatrure; see [1]. However, it is well known that provided some additional conditions, such as a positive lower bound on conjugate radius, the manifolds of (lower) bounded Ricci curvature can be smoothed via various methods (e.g. [10,31,27], etc.) to a nearby metric $g_{\epsilon}$ of bounded sectional curvature. If $(M, g)$ is $\epsilon$-volume collapsed, i.e., the volume of every 1-ball in $(M, g)$ is $\leq \epsilon$, then the injectivity radius of the new metric $\left(M, g_{\epsilon}\right)$ could be also small (e.g. [10]). Therefore a nilpotent Killing structure still exists on such manifolds. Via Ricci flow method ([20]), nilpotent Killing structures are also known to exist on closed manifolds of lower bounded Ricci curvature, whose universal cover satisfies the $(\delta, r)$-Reifenberg condition (see [22]). In particular, Gromov's almost flat manifold theorem and Fukaya's fibration theorem holds $[10,31,22]$. It is a natural question that, besides the original metric, whether those nilpotent Killing structures substantially depend on those different smoothing methods? (see Remark 0.4 below)

The compatibility (resp. stability) of locally constructed pure nilpotent Killing structures (resp. $F$-structures) around neighboring points for one fixed metric, such that one fits inside another, is one of the key steps in construction of a global nilpotent Killing structure (resp. $F$-structure); see [7] and [9]. The stability of pure nilpotent Killing structures associated to a continuous family of metrics $g(t)$ (sufficiently collapsed to a fixed limit) played an important role in solving a continuous version of Klingenberg-Sakai conjecture ([32]).

This paper is devoted to study the stability of pure nilpotent Killing structures for different collapsed metrics on a fixed smooth manifold, and the uniqueness of nilpotent Killing structure obtained by various smoothing methods.

0.2. Main results. Let $d_{L}$ be the Lipschitz distance between two Riemannian metrics on $M$ defined by

$$
d_{L}\left(g_{1}, g_{2}\right)=\inf _{\varphi: M \rightarrow M} \ln \left(\max \left\{\operatorname{dil}(\varphi), \operatorname{dil}\left(\varphi^{-1}\right)\right\}\right)
$$


where the infimum is taken over all diffeomorphisms $\varphi: M \rightarrow M$, and $\operatorname{dil}(\varphi)$ is the optimal Lipschitz constant of $\varphi$ (also called dilatation, cf. [2]). The main result of this paper is as follows.

Theorem 0.1. Given $L_{0}>0, n \geq 1$, there is $\epsilon=\epsilon\left(L_{0}, n\right)>0$ such that the following holds.

Let $g_{i}(i=1,2)$ be two $\epsilon$-collapsed complete Riemannian metrics on a n-manifold $M^{n}$ whose sectional curvature $\left|\sec \left(M, g_{i}\right)\right| \leq 1$. If the nilpotent Killing structure $\mathfrak{n}_{i}$ associated to $g_{i}$ is pure, and

$$
d_{L}\left(g_{1}, g_{2}\right) \leq L_{0},
$$

then there is a diffeomorphism $\Phi: M \rightarrow M$ such that up to a permutation the push-forward $\Phi_{*} \mathfrak{n}_{1}$ is a subsheaf of $\mathfrak{n}_{2}$. If the dimensions of $\mathfrak{n}_{i}$ are the same, then they are isomorphic by $\Phi_{*}$ as sheaves.

Theorem 0.1 improves the local compatibility in [7] and stability of pure nilpotent Killing structures in [32] to a situation that two metrics $g_{1}$ and $g_{2}$ are not close in the Gromov-Hausdorff distance.

Since there are infinitely many pairwise non-conjugate isometric $S^{1}$-actions on $S^{2} \times S^{3}$ (see Example 0.5 below), the collapsing condition in Theorem 0.1 is essential.

Remark 0.2.

(0.2.1) Roughly speaking, the nilpotent structures are determined by the local fundamental group at the same length scale. Condition (0.1.1) is to guarantee the collapsing rates of $g_{i}$ are on a comparable level, such that the subgroups of $\pi_{1}\left(B_{1}(p)\right)$ generated by $g_{i}$-small geodesic loops coincides on each collapsing length scale. (0.2.2) Our construction of $\Phi$ provides a slightly different way from [7] (see Remark 5.5) to prove the local compatibility of pure nilpotent Killing structures on orthogonal fame bundles. By the method in this paper, we are able to construct a global nilpotent structure on an open manifold that only admits a local smoothing (e.g. [27]); see [21].

As a corollary of Theorem 0.1 , we obtain the following uniqueness of pure nilpotent Killing structure by various smoothing techniques.

Theorem 0.3. Given any $n \geq 1, C>0$, there is a positive constant $\epsilon(n, C)>0$ such that for $0<\epsilon<\epsilon(n, C)$, pure nilpotent Killing structures on an $\epsilon$-volume collapsed $n$-manifold $\left(M^{n}, g\right)$ derived by different smoothing methods to a metric $g_{0}$ such that

$$
d_{L}\left(g_{0}, g\right) \leq 1 \text { and }\left|\sec \left(M, g_{0}\right)\right| \leq C
$$

are unique up to a diffeomorphism.

Theorem 0.3 covers the nilpotent Killing structures constructed via Ricci flow in [10, 22] and related evolutions such as [27] on manifolds of bounded Ricci curvature, or via embedding into a Hilbert space in [31] on manifolds of lower bounded Ricci curvature under some additional regularity assumptions (e.g., a positive bound on conjugate radius). 
By Theorem 0.3 again, Theorem 0.1 holds for any $\epsilon$-volume collapsed manifold $(M, g)$ such that there is a nearby metric $g_{0}$ satisfying (0.3.1). For example, if sectional curvature bound in Theorem 0.1 is replaced by

$$
\operatorname{Ric}\left(M, g_{i}\right) \geq-(n-1), \quad \text { conj. } \operatorname{rad}\left(M, g_{i}\right) \geq r_{0}
$$

and $\left(M, g_{i}\right)$ is $\epsilon_{0}\left(L_{0}, n, r_{0}\right)$-volume collapsed, then the nilpotent Killing structure exists [31] and uniquely determined by $g_{i}$, and the stability result in Theorem 0.1 still holds for such $g_{i}$.

In practice, condition (0.1.1) naturally arises in Theorem 0.3 , due to that different methods usually give rise to different curvature bounds.

Indeed, let $C_{i}(t)(i=1,2 ; t \geq 0)$ be universal sectional curvature bounds for two methods $S_{i, t}$ respectively, i.e., $C_{i}(t)=\sup _{g}\left|\sec \left(M, g_{i, t}\right)\right|$, where the supremum is taken over all smoothable metric $g$, and $g_{i, t}=S_{i, t}(g)(i=1,2)$ are smoothed metrics satisfying $d_{L}\left(g_{i, t}, g\right) \leq t$. Then after normalizing to $|\mathrm{sec}| \leq 1$, the rescaled metrics will admit a Lipschitz control

$$
d_{L}\left(C_{1} g_{1, t}, C_{2} g_{2, t}\right) \leq \max \left\{\ln C_{1} / C_{2}, \ln C_{2} / C_{1}\right\}+t, \text { where } C_{i}=C_{i}(t) .
$$

Remark 0.4. We point out the main issues on stability of nilpotent structures constructed by smoothing methods.

First, the general sectional curvature bound $C_{i}(t)$ of $g_{i, t}$ would blow up at a different rate, as $g_{i, t}$ approaches $g$. For an $\epsilon$-volumed collapsed metric $g$, if the ratio $C_{1}(t) / C_{2}(t)$ is large as $t$ small, then the renormalized metrics $C_{i}(t) g_{i, t}$ maybe collapse on different scales.

Secondly, as the underlying metric continuously varies to different scales, subnilpotent structures may appear or vanish several times (see Example 0.5 below). A coherence between nilpotent Killing structures of $g_{1, t}$ and $g_{2, t^{\prime}}\left(t<t^{\prime}\right)$ does not imply the same between $g_{1, t^{\prime}}$ and $g_{2, t^{\prime}}$, as $C_{i}(t) / C_{i}\left(t^{\prime}\right)$ is relative large.

Due to the two issues above, a uniform curvature bound is required in Theorem 0.3 .

For the same reason, the uniqueness in Theorem 0.3 cannot follow from previous results in [7] or [32], where both of them essentially deal with two $C^{1, \alpha}$-close metrics with uniformly bounded curvature.

Here is a typical example that carries different nilpotent Killing structures (cf. [33], [38]).

Example 0.5. Let $S^{2} \times S^{3}$ be endowed which the canonical product metric $h_{0}$. For a positive integer $k$, let $\rho_{k}$ be the isometric free $S^{1}$-action on $S^{2} \times S^{3}$ by

$$
e^{\theta \sqrt{-1}} \cdot(x, z ; v, w)=\left(x, e^{k \theta \sqrt{-1}} z ; e^{\theta \sqrt{-1}} v, e^{\theta \sqrt{-1}} w\right),
$$

where $x$ is real, $z, v, w$ are complex coordinates. By [29, Proposition 5.1], $\rho_{k}$ gives rise to circle bundles of distinct Euler class for different $k$. Thus $\rho_{j}$ and $\rho_{k}(j \neq k)$ are pairwise non-conjugate actions. By shrinking fibers (see [8], cf. [4]) there is a continuous family of Riemannian metrics $g_{k}(\epsilon)$ on $S^{2} \times S^{3}$ collapsing to quotient manifold $S^{2} \times S^{3} / \rho_{k}\left(S^{1}\right)$ with sectional curvature bound $\left|\sec \left(S^{2} \times S^{3}, g_{k}(\epsilon)\right)\right| \leq C$, where $C$ is a constant independent of $k$. 
Thus, the nilpotent Killing structures $\mathfrak{n}_{k}$ of $g_{k}(\epsilon)$ are non-isomorphic, and by joining to $h_{0}$, collapsed metric $g_{1, \epsilon}$ can be changed smoothly to any $g_{k, \epsilon}$.

By Theorem 0.1, the pairwise Lipschitz distance $d_{L}\left(g_{i}(\epsilon), g_{j}(\epsilon)\right)$ goes to $\infty$ for $i \neq j$ as $\epsilon \rightarrow 0$.

In general, let $\mathcal{R}(M ; \epsilon, d)$ be the moduli space endowed with Lipschitz distance, which consists of all isometric classes of $\epsilon$-collapsed Riemannian metrics on a $n$-manifold $M^{n}$, whose sectional curvature $|\sec | \leq 1$ and diameter $\leq d$. Let $\mathcal{R}(M ; \epsilon, d, k)$ be the subspace whose underlying nilpotent Killing structure has dimension equals to $k$. Then by Theorem 0.1 , for $0<\epsilon \leq \epsilon(n, d)$, each component of $\mathcal{R}(M ; \epsilon, d, k)$ corresponds a unique isomorphism class of nilpotent Killing structures on $M$. Moreover, the Lipschitz distance between metrics in $\mathcal{R}(M ; \epsilon, d, k)$ corresponding to distinct nilpotent Killing structures goes to infinity as $\epsilon \rightarrow 0$.

The remaining of the paper is organized as follows. In section 1 we fix some notations, and recall some preliminary facts on submersions and nilpotent Killing structures. Since the proof of Theorem 0.1 is quite long, we first give an outline in section 2. Section 3 to section 6 are devoted to the proof of Theorem 0.1.

Acknowledgment. We owe gratitude to Xiaochun Rong for raising related problems, his constant support and encouragement. The second author is grateful to Fuquan Fang for several highly stimulating conversations. We would like to thank Xuchao Yao for some useful discussions. This work is supported partially by NSFC Grant 11401398 and by Youth Innovative Research Team of Capital Normal University.

\section{Notations AND PRELIMINARIES}

In this section we fix some notations, and recall some elementary facts used later.

1.1. Submersions. Let $f:(M, g) \rightarrow(Y, h)$ be a (not necessarily Riemannian) submersion between two manifolds. The $f$-vertical distribution tangent to $f$-fibers and its orthogonal complement, the $f$-horizontal distribution, are denoted by $\mathcal{V}_{f}$ and $\mathcal{H}_{f}$ respectively. We use $\mathcal{V}_{f}(x)$ (resp. $\mathcal{H}_{f}(x)$ ) to denote the vertical (resp. horizontal) subspace at $x \in M$.

The second fundamental form $I I_{f}$ of $f$-fibers and the integrability tensor $A_{f}$ of $f$ are defined respectively by

$$
\begin{aligned}
I_{f}: \mathcal{V}_{f}(x) \times \mathcal{V}_{f}(x) \rightarrow \mathcal{H}_{f}(x), & I I_{f}(T, T)=\left.\left(\nabla_{T} T\right)^{\perp}\right|_{x}, \\
A_{f}: \mathcal{H}_{f}(x) \times \mathcal{H}_{f}(x) \rightarrow \mathcal{V}_{f}(x), & A(X, Y)=\left.[X, Y]^{\top}\right|_{x} \in \mathcal{V}_{f}(x) .
\end{aligned}
$$

If a submersion $f:(M, g) \rightarrow(Y, h)$ is proper, then $(M, Y, f)$ forms a locally trivial fiber bundle, whose local trivialization can be realized via $f$-horizontal lifting curves. Since our construction of a bundle isomorphism in section 5 relies on this, we recall the local trivialization and related estimates in below.

Let $g$ and $h$ be any fixed Riemannian metric tensor on $M$ and $Y$ respectively. Let $p \in Y$ be a fixed point and let $0<r<\operatorname{inj} \cdot \operatorname{rad}(p)$ in $(Y, h)$. For any point $x \in f^{-1}\left(B_{r}(p)\right)$, there is a unique minimal geodesic $\gamma_{x}:[0,1] \rightarrow Y$ connecting $f(x)=\gamma_{x}(0)$ and $p=\gamma_{x}(1)$. Because $f$ is proper, the horizontal lifting $\tilde{\gamma}_{x}$ : 
$[0,1] \rightarrow M$ at $x$ is uniquely well-defined by $\tilde{\gamma}_{x}(0)=x, f\left(\tilde{\gamma}_{x}(t)\right)=\gamma_{x}(t)$, and tangent vector $\tilde{\gamma}_{x}^{\prime}(t)$ lies in horizontal distribution $\mathcal{H}_{f}$. We define a map

$$
\varphi: f^{-1}\left(B_{r}(p)\right) \rightarrow B_{r}(p) \times F_{p} \quad \text { by } \quad \varphi(x)=\left(f(x), \tilde{\gamma}_{x}(1)\right),
$$

where $F_{p}=f^{-1}(p)$ is a $f$-fiber over $p$.

By construction, $\operatorname{pr}_{1} \circ \varphi=f$, where $\mathrm{pr}_{1}$ is the projection to the 1 st factor. Since $\varphi$ can be viewed as a projection of a flow in $T M$ generated by tangent fields of $\tilde{\gamma}_{x}$ at time $1, \varphi: f^{-1}\left(B_{r}(p)\right) \rightarrow B_{r}(p) \times F_{p}$ is a diffeomorphism. Thus the map $\varphi$ is a local trivialization of fiber bundle $(M, Y, f)$.

Let $\varphi_{2}: f^{-1}\left(B_{r}(p)\right) \rightarrow F_{p}$ be the 2 nd factor of $\varphi$, then by definition

$$
\varphi(x)=\left(f(x), \varphi_{2}(x)\right), \quad \varphi_{2}(x)=\tilde{\gamma}_{x}(1) .
$$

By standard variation methods (cf. Lemma 1 in [25]), $\mathrm{d} \varphi_{2}$ is under control by $I I_{f}, A_{f}$, Lipschitz and co-Lipschitz constant of $f$, and the sectional curvature bound of the base space $Y$, as follows.

Let $L_{0}>0$ be a co-Lipschitz constant of $f$, i.e., for any horizontal vector $\xi \in \mathcal{H}_{f}$, $|\xi|_{g} \leq L_{0} \cdot|\mathrm{d} f(\xi)|_{h}$. Then for any vertical vector $v \in \mathcal{V}_{f}(x)$,

$$
e^{-L_{0} \mid I I r(x)}|v| \leq\left|\mathrm{d} \varphi_{2}(v)\right| \leq e^{L_{0}|I I| r(x)}|v|,
$$

where $|I I|=\sup _{q \in Y}\left|I I_{F_{q}}\right|$, and $r(x)=d(f(x), p) \leq r$ is the distance between points $f(x)$ and $p$.

If $L_{1}$ is a Lipschitz constant of $f$ and $|\sec (Y, h)| \leq 1$, then any horizontal $w \in$ $\mathcal{H}_{f}(x)$ and $0 \leq r(x) \leq \min \left\{\frac{\pi}{2}, r\right\}$,

$$
\left|\mathrm{d} \varphi_{2}(w)\right| \leq C L_{0} L_{1}^{2} e^{L_{0}|I I| r(x)}|A| r(x) \cdot|w|,
$$

where $|A|=\sup _{q \in Y}\left|A_{F_{q}}\right|$ and $C$ is a universal constant.

Now let $f:(M, g) \rightarrow(Y, h)$ be an $\epsilon$-almost Riemannian submersion, i.e., , for any vector $\xi$ perpendicular to a $f$-fiber,

$$
e^{-\epsilon}|\xi|_{g} \leq|\mathrm{d} f(\xi)|_{h} \leq e^{\epsilon}|\xi|_{g} .
$$

By definition and easy calculation, the norms of $I I_{f}$ and $A_{f}$ are pointwisely bounded by the second fundamental form $\nabla^{2} f=\nabla d f$. That is,

$$
\left|I I_{F_{p}}\right| \leq e^{\epsilon} \cdot\left|(\nabla d f)_{F_{p}}\right|, \quad\left|A_{F_{p}}\right| \leq 2 e^{3 \epsilon} \cdot\left|(\nabla d f)_{F_{p}}\right|,
$$

where $|\nabla d f|=\max _{|X|=|Y|=1}|\nabla d f(X, Y)|$.

If $M$ is complete and $Y$ is connected, then for any $p, q \in Y$,

$$
e^{-\epsilon} \cdot d(p, q) \leq d_{H}\left(f^{-1}(p), f^{-1}(q)\right) \leq e^{\epsilon} \cdot d(p, q),
$$

where $d_{H}(A, B)$ is the Hausdorff distance between two subsets $A, B$ in a metric space, i.e., the infimum of $\epsilon>0$ such that the $\epsilon$-neighborhood of $A$ contains $B$ and vice versa.

Indeed, because $f(M)$ is open and close in $Y, f(M)=Y$. By definition, $f$ is $e^{\epsilon}$ Lipschitz, which implies $d(p, q) \leq e^{\epsilon} \cdot d\left(f^{-1}(p), f^{-1}(q)\right)$. Because the horizontal lifting curve of a minimal geodesic $\gamma$ connecting $p$ and $q$ has length $\leq e^{\epsilon} d(p, q)$, $d_{H}\left(f^{-1}(p), f^{-1}(q)\right) \leq e^{\epsilon} \cdot d(p, q)$. 
The injectivity radius function inj. $\operatorname{rad}_{g}: M \rightarrow \mathbb{R}$ on a complete Riemannian manifold $(M, g)$ is known to be locally 1-Lipschitz [39], i.e., for any two points $p, q$ in $M$,

$$
\text { inj. } \operatorname{rad}_{g}(q) \geq \min \left\{\text { inj. } \operatorname{rad}_{g}(p), \operatorname{conj} \cdot \operatorname{rad}_{g}(q)\right\}-d(p, q),
$$

where conj. $\operatorname{rad}_{g}(q)$ is the conjugate radius at $q$, and $d(p, q)$ is the distance between $p$ and $q$.

Given real numbers $L \geq 1$ and $\epsilon \geq 0$, a (not necessarily continuous) map $\psi$ : $X \rightarrow Y$ between metric spaces is called a $(L, \epsilon)$-quasi-isometry if for all $x_{1}$ and $x_{2}$ in $X$,

$$
L^{-1} d_{X}\left(x_{1}, x_{2}\right)-\epsilon \leq d_{Y}\left(\psi\left(x_{1}\right), \psi\left(x_{2}\right)\right) \leq L d_{X}\left(x_{1}, x_{2}\right)+\epsilon,
$$

and $\psi(X)$ is $\epsilon$-dense in $Y$.

We use $\varkappa(\epsilon \mid a, b, c, \ldots)$ to denote a positive function depending on $\epsilon, a, b, c, \ldots$ such that after fixing $a, b, c, \ldots, \varkappa(\epsilon \mid a, b, c, \ldots) \rightarrow 0$ as $\epsilon \rightarrow 0$. It will be simply written as $\varkappa(\epsilon)$, if the dependence is clear.

1.2. Nilpotent Killing structures. The reference for this subsection is [7].

Let $\mathfrak{n}$ be a sheaf of Lie algebras generated by locally defined smooth vector fields on $M$. A metric $g$ on $M$ is called $\mathfrak{n}$-invariant, if all (local) sections of $\mathfrak{n}$ are Killing fields for $g$.

For a local section $X$ of $\mathfrak{n}$, its flow defines a local one-parameter action. A set $Z \subset M$ is called invariant if $Z$ is preserved by all such actions. For any point $p \in M$, the orbit $O_{p}$ of $p$ is defined to be the minimal invariant set containing $p$.

Let $\mathfrak{n}$ be a sheaf of nilpotent Lie algebras. It is called a nilpotent Killing structure for $g$, if for any $p \in M$, there is an invariant neighborhood $U$ of $p$ and a normal covering $\pi: \tilde{U} \rightarrow U$ such that

(1) The integral of the pullback sheaf $\pi^{*} \mathfrak{n}(\tilde{U})$ generates an isometric action $\rho$ of a simply connected nilpotent Lie group $N_{U}$, whose kernel $K=\operatorname{ker} \rho$ is discrete.

(2) $N_{U}$ and the deck-transformation group $\Lambda$ on $\tilde{U}$ generates an isometric action of a Lie group $H$ of finite many components, extending that of $\Lambda$ such that the identity component $N_{0}=N_{U} / K$.

(3) For any open $\tilde{W} \subset \tilde{U}$ containing a preimage point of $p$, the structure homomorphism $\pi^{*} \mathfrak{n}(\tilde{U}) \rightarrow \pi^{*} \mathfrak{n}(\tilde{W})$ is an isomorphism.

(4) The neighborhood $U$ and covering $\tilde{U}$ can be chosen independent of $p \in O_{p}$. The $\mathfrak{n}$-invariant metric $g$ is called $(\rho, k)$-round, if in addition, the neighborhood $U$ above can be chosen to satisfies the following properties.

(1) $U$ contains a metric ball $B_{\rho}(p)$ and all points in $\tilde{U}$ away from boundary have injectivity radius $>\rho$.

(2) $\# H / N_{0}=\# \Lambda /\left(\Lambda \cap N_{0}\right) \leq k$.

To illustrate what happens, we give some elementary but typical examples.

Let $N$ be a simply connected nilpotent Lie group, $\Lambda$ be a co-compact discrete subgroup, and let $g$ be a left invariant metric on $N$. Let $\mathfrak{n}$ be the sheaf of right invariant vector fields, which are Killing fields for $g$. 
Example 1.9 (nilmanifolds). Let $X$ be a right invariant vector field on $N$. Then for any $a \in N$ and $\lambda \in \Lambda, \lambda \cdot \exp t X \cdot a=\exp t \operatorname{Ad}_{\lambda} X \cdot \lambda \cdot a$. The conjugate quotient $\mathfrak{n}$ by $\Lambda$ defines a canonical nilpotent Killing structure on the nilmanifold $\Lambda \backslash N$. The center of $\mathfrak{n}$ will descend to a subsheaf which generates a torus action on $\Lambda \backslash N$.

Let $\nabla^{\text {can }}$ be the canonical flat connection with parallel torsion on $N$. Then its affine transformation group is $N \rtimes \operatorname{Aut}(N)$. A infra-nilmanifold $Z$ is a compact quotient manifold $\Gamma \backslash N$, where $\Gamma$ is a discrete subgroup of $N \rtimes \operatorname{Aut}(N)$. The connection $\nabla^{\text {can }}$ descends to $Z$, which is called a canonical affine structure. The affine group is denoted by $\operatorname{Aff}(\Gamma \backslash N)$. Since the index $[\Gamma: \Gamma / \Gamma \cap N]<w(n)$, the canonical nilpotent Killing structure on $(\Gamma \cap N) \backslash N$ induces a canonical nilpotent Killing structure on $Z$.

Example 1.10 (affine bundles). A fiber bundle $(X, Y, f)$ is called to be affine, if its fiber $Z$ is diffeomorphic to a infra-nilmanifold $\Lambda \backslash N$ and its structure group is contained in $\operatorname{Aff}(\Gamma \backslash N)$. The sheaf of parallel vector fields along fibers naturally form a nilpotent Killing structure $\mathfrak{n}$ on $X$ (cf. [7, II.4]). A metric $g$ on $X$ is called affine-invariant, if it is $\mathfrak{n}$-invariant.

Let $G$ be a compact group acting on $X$ and $Y$ isometrically. If the bundle projection $f$ is $G$-equivariant, i.e.,

$$
f(g \cdot x)=g \cdot f(x), \quad \text { for } \forall g \in G, \forall x \in X,
$$

and at the same time, $G$ preserves the affine structure on every fiber, then the action of $G$ extends to an action on $\mathfrak{n}$, such that the actions of $\mathfrak{n}$ and $G$ on $X$ commute (see [7] for details). If the action of $G$ is free, then the quotient sheaf $\overline{\mathfrak{n}}$ on $X / G$ is also a nilpotent Killing structure.

A bundle map $\Phi$ between two affine bundles $\left(X_{i}, Y_{i}, f_{i}\right)(i=1,2)$ is called affineequivariant, if it preserves the affine structures, i.e., $\Phi_{*} \mathfrak{n}_{1}$ is a subsheaf of $\mathfrak{n}_{2}$.

The existence of a nilpotent Killing structure was proved in [7] for collapsed manifolds with bounded sectional curvature.

Theorem 1.11 ([7]). For any $\delta>0$ and integer $n>0$, there are $\rho, \epsilon>0$, and integer $k \geq 1$ such that the following holds.

Let $(M, g)$ be an $\epsilon$-collapsed $n$-manifold of $|\sec (M, g)| \leq 1$. Then $M$ carries a nilpotent Killing structure $\mathfrak{n}$ of positive rank for a nearby $\mathfrak{n}$-invariant metric $g_{\delta}$, which is $(\rho, k)$-round and satisfies

(1) $g^{-\delta} g<g_{\epsilon}<e^{\delta} g$,

(2) $g_{\delta}$ 's connection is $\varkappa(\delta)$-close to that of $g$,

(3) curvature operator $R_{g_{\delta}}$ and its $i$-th covariant derivatives is bounded by $c(n, i, \delta)$.

By its construction in [7], $\mathfrak{n}$ is induced by an $O(n)$-invariant nilpotent Killing structure on an $O(n)$-equivariant affine bundle $(F M, Y, f)$, where $F M$ is the orthonormal frame of $M$ with a canonical metric induced by a bi-invariant metric on $O(n)$ and the Levi-Civita connection of $g$.

Conversely, let $\mathfrak{n}$ be a nilpotent Killing structure on $(M, g)$ and $g$ is $\mathfrak{n}$-invariant. Then by definition, the differential of local actions of $\mathfrak{n}$ gives rise to a nilpotent 
Killing structure $\tilde{\mathfrak{n}}$ on $F M$, which corresponds to a canonical nilpotent structure on an $O(n)$-equivariant affine bundle, whose quotient is $\mathfrak{n}$.

We call two fiber bundles $\left(X_{i}, Y_{i}, f_{i}\right)(i=1,2)$ to be isomorphic if there are diffeomorphisms $\Phi: X_{1} \rightarrow X_{2}$ and $\Psi: Y_{1} \rightarrow Y_{2}$ such that $\Psi \circ f_{1}=f_{2} \circ \Phi$. Two affine bundles are isomorphic if such $\Phi$ also preserves the affine structure. Clearly, $\Phi$ is affine if and only if $\Phi$ is $\mathfrak{n}$-equivariant, i.e., $\Phi$ commutes with the local actions of the canonical nilpotent Killing structure.

\section{OUTLINE OF PROOF THEOREM 0.1}

Our main Theorem 0.1 is a consequence of the following.

Theorem 2.1. Given any real number $L_{0} \geq 1, \delta_{0}>0$, and positive integers $n>$ $m \geq 1$, there is $\epsilon_{0}\left(L_{0}, \delta_{0}, n\right)>0$ (independent of $\lambda \geq 1$ ) such that the following holds.

Let $M^{n}$ be a complete $n$-manifold and $\left(M^{n}, Y_{i}^{m}, f_{i}\right)(i=1,2)$ be affine bundles over m-manfolds $Y_{i}^{m}$ respectively. Let $g_{i}$ be an affine-invariant (w.r.t. $f_{i}$ ) Riemannian metric on $M$ such that

$$
|\sec (M, g)| \leq 1, \quad\left|\sec \left(Y_{i}, h_{i}\right)\right| \leq 1,
$$

where $h_{i}$ is the quotient metric of $g_{i}$ on $Y_{i}$. Assume that

(2.1.2) the metrics $g_{1}, g_{2}$ are $L_{0}$-equivalent, i.e., $L_{0}^{-1} g_{2} \leq g_{1} \leq L_{0} g_{2}$, and

(2.1.3) for any $p \in Y_{i}$, the diameter and the second fundamental form of $f_{i}$-fiber $F_{i, p}=f_{i}^{-1}(p)$ satisfies

$$
\operatorname{diam}_{g_{i}} F_{i, p} \leq \epsilon \cdot \min \left\{1, \text { inj. } \operatorname{rad}_{h_{i}}(p)\right\}, \quad\left|I_{F_{i, p}}\right| \leq \delta_{0} .
$$

Then there is an affine bundle isomorphism $(\Phi, \Psi)$ such that $f_{2} \circ \Phi=\Psi \circ f_{1}$.

If in addition, there is a Lie group $G$ acting isometrically on both of $\left(M, g_{i}\right)$ and $\left(Y_{i}, h_{i}\right)$ so that $f_{i}$ is a $G$-equivariant affine bundle, then the diffeomorphisms in the bundle isomorphism between $\left(M, Y_{i}, f_{i}\right)$ are also $G$-equivariant.

Note that, in Theorem 2.1 we do not assume $f_{i}$-fibers absorb all collapsed directions. Hence, potentially $Y_{i}$ maybe also collapse.

Compared to the earlier stability results ([7, section 7], [23], [36], [25], etc.), our main improvement here is that no fiberwise closeness (nor $C^{1}$-closeness) of $f_{i}$ are required.

Theorem 0.1 is a corollary of Theorem 2.1. Indeed, by (0.1.1), up to a shift of the collapsing scale, the nilpotent Killing structure $\mathfrak{n}_{i}(i=1,2)$ corresponding to $g_{i}$ can be chosen of the same dimension. According to [7] (or see section 2.2), there is a complete Riemannian manifold $Y_{i}$ and an $O(n)$-invariant affine bundle, $\left(F M, Y_{i}, \tilde{f}_{i}\right)$, on the orthogonal frame bundle $F M$, whose canonical nilpotent Killing structure descends $O(n)$-equivariantly to $\mathfrak{n}_{i}$ on $\left(M, g_{i}\right)$. Since there are nearby $\mathfrak{n}_{i}$-invariant metrics $g_{i, \epsilon}$ of uniformly bounded sectional curvature, without loss of generality we assume that $g_{i}$ (resp. the induced metric $\tilde{g}_{i}$ on $F M$ ) itself is $\mathfrak{n}_{i^{-}}$ invariant (resp. $O(n)$-invariant and $\mathfrak{n}_{i}$-invariant). Thus Theorem 0.1 is reduced to 
the stability of affine bundles with invariant metrics. Let $\tilde{\Phi}$ be the affine bundle isomorphism between $\left(F M, Y_{i}, \tilde{f}_{i}\right)$ provided by Theorem 2.1. Then its $O(n)$-quotient is the desired diffeomorphism in Theorem 0.1.

The main part of this paper is devoted to the proof of Theorem 2.1, which is divided into three steps:

Step 1. construct a $e^{\epsilon} L_{0}$-bi-Lipschitz diffeomorphism $\Psi: Y_{1} \rightarrow Y_{2}$ such that $d\left(\Psi \circ f_{1}, f_{2}\right) \leq 2 L_{0} \epsilon$. See Proposition 4.1.

Step 2. construct a diffeomorphism $\Phi_{1}: M \rightarrow M$ such that $\Phi_{1}$ is $2 L_{0}^{2} \epsilon$-close to $\operatorname{Id}_{M}$ (measured in $g_{1}$ ), $f_{2}=\Psi \circ f_{1} \circ \Phi_{1}$, and for any $p \in Y_{1}$ and $F_{1, p}=f_{1}^{-1}(p)$, the restriction $\left.\Phi_{1}\right|_{F_{2,}}: F_{2, p} \rightarrow F_{1, \Psi(p)}$ is $e^{\epsilon} L_{0}$-bi-Lipschitz. See Proposition 5.3.

Step 3. modify $\Phi_{1}$ to get a bundle isomorphism $\Phi_{2}: M \rightarrow M$ that preserves the affine bundle structure. See Proposition 6.2.

If in addition, $f_{i}$ is $G$-equivariant, where $G$ acts by isometries, then so are $\Phi_{1}$, $\Phi_{2}$ and $\Psi$.

The key in first two steps is a weak $C^{1}$-closeness between $f_{i}$ in the following sense, whose proof will be carried out in Section 3.

Proposition 2.2 (Weak $C^{1}$-closeness of $\left.f_{i}\right)$. Let $f_{i}:\left(M^{n}, g_{i}\right) \rightarrow\left(Y_{i}^{m}, h_{i}\right)(i=1,2)$ be two $\epsilon$-Riemannian submersions satisfying (2.1.1), (2.1.2), (2.1.3) and $\left|\nabla^{2} f_{i}\right| \leq$ $\delta_{0}(i=1,2)$. Then the followings hold.

(2.2.1) For any $x \in M$, the dihedral angle measured in $g_{i}$ between vertical subspaces $\mathcal{V}_{f_{1}}(x)$ and $\mathcal{V}_{f_{2}}(x) \leq \varkappa\left(\epsilon \mid L_{0}, \delta_{0}, n\right)$.

(2.2.2) For any $f_{1}$-horizontal vector $w \in T_{x} M$ with $|w|_{g_{1}}=1$,

$$
e^{-\varkappa\left(\epsilon \mid L_{0}, \delta_{0}, n\right)} L_{0}^{-1} \leq\left|d f_{2}(w)\right| \leq e^{\varkappa\left(\epsilon \mid L_{0}, \delta_{0}, n\right)} L_{0} .
$$

For any $p \in Y_{1}$, after fixing a point $x \in f_{1}^{-1}(p)$, a smooth map $\psi_{p, x}$ can be defined by

$$
\psi_{p, x}: B_{r}(p) \rightarrow Y_{2}, \quad q \mapsto \psi_{p, x}(q)=f_{2}\left(\tilde{\gamma}_{q}(1)\right),
$$

where $0<r<\operatorname{inj} \cdot \operatorname{rad}_{h_{1}}(p)$, and $\tilde{\gamma}_{q}(t):[0,1] \rightarrow M$ is the $f_{1}$-horizontal lifting at $x$ of the unique minimal geodesic $\gamma_{q}:[0,1] \rightarrow Y_{1}$ from $p$ to $q$. By Proposition 2.2, $\Psi_{p, x}$ is a $L_{0} e^{\varkappa\left(\epsilon \mid L_{0}, \delta_{0}, n\right)}$-bi-Lipschitz diffeomorphism ${ }^{1}$ from $B_{r}(p)$ onto an open set $V \subset Y_{2}$; see Remark 5.6 below. The map $\Psi$ is essentially an average of local diffeomorphisms $\left\{\psi_{p, x}\right\}_{x \in f_{1}^{-1}(p)}$; see Section 4.

Our construction of $\Phi_{1}$ in Section 5 is via $f_{1}$-horizontal liftings of minimal geodesics, which is different from [7, Proposition A.2.2] by the normal projection on fibers through minimal geodesics. It requires only weak $C^{1}$-closeness and $C^{0}$ closeness of $\Psi \circ f_{1}$ and $f_{2}$. The method in [7] still works with some additional arguments to guarantee $\Psi \circ \tilde{f}_{1}$ and $f_{2}$ to be $C^{1}$-close; see Remark 4.2.

In Section 6, we will further prove that, after identifying the simply connected nilpotent groups associated to $\mathfrak{n}_{i}$ by their lattice, their actions on the universal cover of local neighborhoods of points in $M$ are $C^{1}$-close; see Lemma 6.1. Then $\Phi_{2}$ is

\footnotetext{
${ }^{1}$ This property roots back to an observation by Xiang Li and Xiaochun Rong, see [26].
} 
obtained by the same average method in [7], i.e., averaging over a infra-nil fiber so that actions of the corresponding nilpotent group are conjugate on a local cover.

\section{WEAK $C^{1}$-CLOSENESS}

In this section we prove Proposition 2.2. Let $f_{i}:\left(M^{n}, g_{i}\right) \rightarrow\left(Y_{i}^{m}, h_{i}\right)(i=$ $1,2)$ be two $\epsilon$-almost Riemannian submersions which satisfy $\left|\sec \left(M, g_{i}\right)\right| \leq 1$, $\left|\sec \left(Y_{i}, h_{i}\right)\right| \leq 1$ and the following three conditions:

(3.1.1) $g_{i}$ are $L_{0}$-equivalent, i.e., $L_{0}^{-1} g_{2} \leq g_{1} \leq L_{0} g_{2}$.

(3.1.2) for any $p \in Y_{i}$, the intrinsic diameter of the $f_{i}$-fiber $F_{i, p}=f_{i}^{-1}(p)$ satisfies $\operatorname{diam}_{g_{i}} F_{i, p} \leq \epsilon \cdot \min \left\{1\right.$, inj. $\left.\operatorname{rad}_{h_{i}}(p)\right\}$.

(3.1.3) the second fundamental form of $f_{i}$ satisfies $\left|\nabla^{2} f_{i}\right|_{C^{0}} \leq \delta$.

Note that no uniformly injectivity radius on $\left(Y_{i}, h_{i}\right)$ is assumed, and a prior there is no bound between inj. $\operatorname{rad}_{h_{1}}\left(f_{1}(x)\right)$ and inj. $\operatorname{rad}_{h_{2}}\left(f_{2}(x)\right)$ for a point $x \in M$.

A key observation is that, after lifting $f_{i}$ to the iterated tangent space $T_{o}\left(T_{x} M\right)$ and blowing up the pull-back metrics, they would be close to two linear maps respectively, such that up to a diffeomorphic chart transformation, their fibers coincide with each other.

The proof is based on a quasi-isometry $\psi:\left(Y_{1}, h_{1}\right) \rightarrow\left(Y_{2}, h_{2}\right)$ such that $\psi \circ f_{1}$ is close to $f_{2}$, which naturally defined by a shift between $f_{i}:\left(M, g_{i}\right) \rightarrow\left(Y_{i}, h_{i}\right)$ $(i=1,2)$ below.

For $p \in Y_{1}$, let us define $\psi(p)$ to be a point in $f_{2}\left(f_{1}^{-1}(p)\right)$. Then by (3.1.1), for any $x \in M$ and $p=f_{1}(x)$,

$$
d\left(\psi\left(f_{1}(x)\right), f_{2}(x)\right) \leq e^{\epsilon} \cdot \operatorname{diam}_{g_{2}} F_{1, p} \leq e^{\epsilon} L_{0} \operatorname{diam}_{g_{1}} F_{1, p} .
$$

Moreover, it is easy to see that

$$
d(\psi(p), \psi(q)) \leq e^{\epsilon} L_{0} d_{H, g_{1}}\left(F_{1, p}, F_{1, q}\right)+e^{\epsilon} L_{0} \operatorname{diam}_{g_{1}} F_{1, p}, \quad \text { and }
$$

$$
d(\psi(p), \psi(q)) \geq e^{-\epsilon} L_{0}^{-1} d_{H, g_{1}}\left(F_{1, p}, F_{1, q}\right)-e^{\epsilon} \operatorname{diam}_{g_{2}} F_{2, p}-e^{\epsilon} L_{0} \operatorname{diam}_{g_{1}} F_{1, p} .
$$

Since by (1.4.3), $d_{H, g_{1}}\left(F_{1, p}, F_{1, q}\right)$ is proportional to $d(p, q)$ by $e^{\epsilon}$, we have

Lemma 3.3. The map $\psi$ is an $\left(e^{2 \epsilon} L_{0}, 2 e^{\epsilon} L_{0} \epsilon\right)$-quasi-isometry.

Before given the proof of Proposition 2.2, we make some preparation.

Let us fix a point $x \in M$ and consider the exponential map of $\left(M, g_{2}\right), \exp _{x ; g_{2}}$ : $T_{x} M \rightarrow M$. Let $g_{1}^{*}=\exp _{x ; g_{2}}^{*}\left(g_{1}\right)$ and $g_{2}^{*}=\exp _{x ; g_{2}}^{*}\left(g_{2}\right)$ be the pullback metric tensors on $T_{x} M$, and pull back again $g_{i}^{*}$ on $T_{x} M$ to the tangent space $T_{o}\left(T_{x} M\right)$ by $\exp _{o ; g_{1}^{*}}$ of $g_{1}^{*}$, where the pullback tensors are denoted by $g_{i}^{* *}$.

Then the ball $B_{\frac{\pi}{2}}\left(o ; g_{1}^{*}\right) \subset\left(T_{o}\left(T_{x} M\right),\left.g_{1}^{*}\right|_{o}\right)$, denoted again by $U$, satisfies (cf. [39])

$$
\text { inj. } \operatorname{rad}\left(U, g_{i}^{* *}\right) \geq \frac{\pi}{2} L_{0}^{-1}, \quad\left|\sec \left(U, g_{i}^{* *}\right)\right| \leq 1 .
$$

The lifting $\epsilon$-almost Riemannian submersions are well-defined on $U$,

$$
\tilde{f}_{i}=f_{i} \circ \exp _{x ; g_{2}} \circ \exp _{o ; g_{1}^{*}}:\left(U, g_{i}^{* *}\right) \rightarrow \tilde{f}_{i}(U) \subset\left(Y_{i}, h_{i}\right) .
$$


Let $\left\{f_{i, j}:\left(M_{j}, g_{i, j}\right) \rightarrow\left(Y_{i, j}, h_{i, j}\right)\right\}_{i=1,2}$ be a contradiction sequence to Proposition 2.2 with $\epsilon_{j} \rightarrow 0$, where the conclusion fails at a point $x_{j} \in M_{j}$. Without loss of generality, we assume that inj. $\operatorname{rad}_{h_{1}}\left(f_{1, j}(x)\right) \leq$ inj. $\operatorname{rad}_{h_{2}}\left(f_{2, j}(x)\right)$. Let $\hat{\varepsilon}_{j}=\epsilon_{j}^{1 / 2} \cdot \operatorname{inj} . \operatorname{rad}_{h_{1}}\left(f_{1}(x)\right)$.

After blowing up with $\hat{\varepsilon}_{j}^{-1}$, by Cheeger-Gromov convergence theorem $([5,6,18]$, cf. [16, 30, 24]) and (3.3.1),

$$
\begin{aligned}
&\left(U_{j}, o_{j}, \hat{\varepsilon}_{j}^{-2} g_{i, j}^{* *}\right) \stackrel{C^{1, \alpha}}{\longrightarrow}\left(\mathbb{R}^{n}, g_{i, \infty}, o_{i}\right), j \rightarrow \infty, \\
&\left(Y_{i, j}, f_{i, j}\left(x_{j}\right), \hat{\varepsilon}_{j}^{-2} h_{i, j}\right) \stackrel{C^{1, \alpha}}{\longrightarrow}\left(\mathbb{R}^{m}, o_{i}\right), \quad j \rightarrow \infty .
\end{aligned}
$$

Then the identity map $I_{j}:\left(U_{j}, o_{j}, \hat{\varepsilon}_{j}^{-2} g_{1, j}^{* *}\right) \rightarrow\left(U_{j}, o_{j}, \hat{\varepsilon}_{j}^{-2} g_{2, j}^{* *}\right)$ converges to a smooth bi-Lipschitz map $I_{\infty}:\left(\mathbb{R}^{n}, g_{1, \infty}, o_{1}\right) \rightarrow\left(\mathbb{R}^{n}, g_{2, \infty}, o_{2}\right)$, which in general is not linear.

By passing to a subsequence, the lifting map defined by (3.3.2),

$$
\tilde{f}_{i, j}:\left(U_{j}, o_{j}, \hat{\varepsilon}_{j}^{-2} g_{i, j}^{* *}\right) \rightarrow\left(\tilde{f}_{i, j}\left(U_{j}\right), f_{i, j}\left(x_{j}\right), \hat{\varepsilon}_{j}^{-2} h_{i, j}\right),
$$

converges to a canonical projection $\tilde{f}_{i, \infty}:\left(\mathbb{R}^{n}, g_{i, \infty}, o\right) \rightarrow\left(\mathbb{R}^{m}, o_{i}\right)$.

Let us consider the quasi-isometry $\psi_{j}: Y_{1, j} \rightarrow Y_{2, j}$ defined for $\left\{f_{i, j}\right\}$ such that $\psi_{j}$ maps $f_{1, j}\left(x_{j}\right)$ to $f_{2, j}\left(x_{j}\right)$. By (3.2.1) and after blow-up, the distance error measured on $\left(Y_{2, j}, \hat{\varepsilon}_{j}^{-2} h_{2, j}\right)$ satisfies that, for any $\tilde{y} \in U_{j}$,

$$
d\left(\psi_{j} \circ \tilde{f}_{1, j}(\tilde{y}), \tilde{f}_{2, j}(\tilde{y})\right) \leq e^{\epsilon_{j}} L_{0} \cdot \hat{\varepsilon}_{j}^{-1} \operatorname{diam}_{g_{1, j}} f_{1, j}^{-1}\left(\tilde{f}_{1, j}(\tilde{y})\right) .
$$

Moreover, for any $p, q \in\left(Y_{1, j}, \hat{\varepsilon}_{j}^{-2} h_{1, j}\right)$, we derive from (3.2.2) that

$$
\begin{aligned}
& d\left(\psi_{j}(p), \psi_{j}(q)\right) \\
\leq & e^{2 \epsilon_{j}} L_{0} \cdot d(p, q)+e^{2 \epsilon_{j}} L_{0} \cdot \hat{\varepsilon}_{j}^{-1} \cdot \min \left\{\operatorname{diam}_{g_{1, j}} f_{1, j}^{-1}(p), \operatorname{diam}_{g_{1, j}} f_{1, j}^{-1}(q)\right\} .
\end{aligned}
$$

By the choice of $\hat{\varepsilon}_{j}$ and $\left|\nabla^{2} f_{i, j}\right| \leq \delta_{0}$,

$$
\hat{\varepsilon}_{j}^{-1} \cdot \operatorname{diam}_{g_{1, j}} f_{1, j}^{-1}\left(\tilde{f}_{1, j}(\tilde{y})\right) \leq \sqrt{\epsilon_{j}} .
$$

It follows from a standard diagonal procedure that a subsequence of $\psi_{j}$ converges to a $L_{0}$-Lipschitz map $\psi_{\infty}:\left(\mathbb{R}^{n}, o_{1}\right) \rightarrow\left(\mathbb{R}^{n}, o_{2}\right)$ such that

$$
\phi_{\infty} \circ \tilde{f}_{1, \infty}=\tilde{f}_{2, \infty} \circ I_{\infty} \text {. }
$$

We are now ready to prove Proposition 2.2.

Proof of Proposition 2.2. Recall that the dihedral angle between the vertical subspaces $\mathcal{V}_{f_{1}}(x)$ and $\mathcal{V}_{f_{2}}(x)$ is defined to be the Hausdorff distance $d_{H}\left(S V_{f_{1}}(x), S V_{f_{2}}(x)\right)$ in the $g_{2}$-unit sphere of $T_{x} M$, where $S V_{f_{i}}(x)=\mathcal{V}_{f_{i}} \cap S_{x} M$ and $S_{x} M$ is the $g_{2^{-}}$ unit sphere centered at the origin of $T_{x} M$. Because $g_{1}$ and $g_{2}$ are $L_{0}$-equivalent, it makes no substantial difference, if instead, the dihedral angle is measured in $g_{1}$. 
Since $\left|\nabla^{2} f_{i}\right| \leq \delta_{0}$, it is clear that the contradicting sequence above is $\varkappa(\epsilon)$-close to $\tilde{f}_{i, \infty}$ in the $C^{1, \alpha}$-norm, whose fibers, by (3.3.3), coincide with each other. So by a contradiction argument, we derive (2.2.1).

Since the pullback metric $I_{\infty}^{*} g_{2, \infty}$ satisfies

$$
L_{0}^{-1} I_{\infty}^{*} g_{2, \infty} \leq g_{1, \infty} \leq L_{0} I_{\infty}^{*} g_{2, \infty}
$$

by (3.3.3), for any $\tilde{f}_{1, \infty}$-horizontal vector $w \in T_{o} \mathbb{R}^{m}$ with $|w|_{g_{1, \infty}}=1$,

$$
L_{0}^{-1} \leq\left|\mathrm{d} \tilde{f}_{2, \infty}(w)\right| \leq L_{0} .
$$

By $C^{1, \alpha}$-convergence of $\tilde{f}_{i, j}$ again, (2.2.2) follows from a contradiction argument.

Remark 3.4. Clearly, (2.2.2) implies a uniform control on the deviation of $f_{i}$-horizontal distributions from each other. However, they are not necessarily close. An easy example can be found on a flat torus, where the two metrics $g_{1}$ and $g_{2}$ are induced from $\left(\mathbb{R}^{2}, \tilde{g}_{i}\right)$ with two flat metrics whose orthonormal decompositions are different from each other by a definite angle.

\section{DifFeOmorphism $\Psi$ Between Base Spaces}

We are to improve the quasi-isometry $\psi$ in Lemma 3.3 to a bi-Lipschitz diffeomorphism $\Psi: Y_{1} \rightarrow Y_{2}$ via center of mass.

Let $(F, v)$ be a probability measure space and let $\imath: F \rightarrow Y_{2}$ be a measurable map into $\left(Y_{2}, h_{2}\right)$. If its image $t(F)$ is contained in a convex ball $B_{a}(z)$ of radius $a<\frac{\pi}{6}$, then the smooth energy function

$$
E(y)=\frac{1}{2} \int_{F} d^{2}(l(x), y) d v
$$

is strictly convex in $B_{3 a}(z)$. It is clear that $E$ takes a unique minimum point at some point $z_{1}$ in the closure of $B_{2 a}(z)$. We call $z_{1}$ the center of mass of $\iota$ (cf. [19], [7]).

Proposition 4.1. There is $\epsilon_{0}=\epsilon_{0}\left(L_{0}, \delta_{0}, n\right)>0$ such that for any two $\epsilon$-almost Riemannian submersions $f_{i}:\left(M, g_{i}\right) \rightarrow\left(Y_{i}, h_{i}\right)$ in Proposition 2.2 with $\epsilon<\epsilon_{0}$, there is a $e^{\varkappa\left(\epsilon \mid L_{0}, \delta_{0}, n\right)} L_{0}$-bi-Lipschitz diffeomorphism $\Psi:\left(Y_{1}, h_{1}\right) \rightarrow\left(Y_{2}, h_{2}\right)$ satisfying that, for any $p \in Y_{1}$ and $x \in F_{1, p}=f_{1}^{-1}(p)$,

$$
d\left(\Psi \circ f_{1}(x), f_{2}(x)\right) \leq 2 L_{0} \operatorname{diam}_{g_{1}} F_{1, p} .
$$

If in addition, $f_{i}(i=1,2)$ are $G$-equivariant, then $\Psi$ is also $G$-equivariant.

Proof. Since the diameter of $f_{2}\left(F_{1, p}\right)$,

$$
\operatorname{diam}_{h_{2}}\left(f_{2}\left(F_{1, p}\right)\right) \leq L_{0} e^{\epsilon} \epsilon<\frac{\pi}{6},
$$

let us define $\Psi(p)$ to be the center of mass of $f_{2}: F_{1, p} \rightarrow\left(Y_{2}, h_{2}\right)$. By definition, (3.2.1) implies (4.1.1). In the following we prove that $\Psi$ is a diffeomorphism. 
By definition, $\Psi(p)$ is the critical point of the energy functional $E_{p}(\cdot)=E(p ; \cdot)$, where

$$
\begin{aligned}
E(p ; y) & =\frac{1}{2} f_{F_{1, p}} d^{2}\left(f_{2}(x), y\right) \operatorname{dvol}(x) \\
& =\frac{1}{2 \operatorname{vol}\left(F_{1, p}\right)} \int_{F_{1, p}} d^{2}\left(f_{2}(x), y\right) \operatorname{dvol}(x)
\end{aligned}
$$

Let $\left(p^{1}, p^{2}, \ldots, p^{m}\right)$ and $\left(y^{1}, y^{2}, \cdots, y^{m}\right)$ be local coordinates around $p$ and $\Psi(p)$ respectively. Then $y=\Psi(p)$ is the implicit function determined by the Pfaffian equation, $\partial_{y} E(p ; y)=0$, a system of equations in local coordinates on $Y_{1} \times Y_{2}$ below:

$$
\begin{aligned}
& E_{j}^{\prime}\left(p^{1}, \ldots, p^{m} ; y^{1}, \ldots, y^{m}\right) \\
= & f_{F_{1, p}} d\left(f_{2}(x), y\right)\left\langle\nabla r_{f_{2}(x)}, \frac{\partial}{\partial y^{j}}\right\rangle \operatorname{dvol}(x)=0, \quad(j=1, \ldots, n)
\end{aligned}
$$

where $r_{y}=d(y, \cdot)$ be the distance function on $\left(Y_{2}, h_{2}\right)$.

Let $E^{\prime}=\left(E_{1}^{\prime}, \ldots, E_{m}^{\prime}\right)$ and $\partial_{y} E^{\prime}$ be the differential of $E^{\prime}(p ; \cdot)$ after fixing $p$. Then it is easy to determine $d \Psi$ by

$$
d \Psi=-\left(\partial_{y} E^{\prime}\right)^{-1} \circ \partial_{p} E^{\prime},
$$

As for $\partial_{p} E^{\prime}$, we may assume that $\left(y^{1}, \ldots, y^{m}\right)$ is the normal coordinates at $y \in$ $\left(Y_{2}, h_{2}\right)$. Then by identifying points and their position vectors,

$$
d\left(f_{2}(x), y\right) \nabla r_{f_{2}(x)}=-f_{2}(x) .
$$

Thus (4.1.3) can be rewritten as a vector equation

$$
E^{\prime}\left(p^{1}, \ldots, p^{m} ; 0, \ldots, 0\right)=f_{F_{1, p}}-f_{2}(x) \operatorname{dvol}(x)=0 .
$$

For any unit-speed geodesic $\gamma(t)$ with $\gamma(0)=p$, let $x(t) \in F_{1, \gamma(t)}$ be its $f_{1}$-horizontal lifting that starts at $x \in F_{1, p}$. By direct calculation,

$$
\begin{aligned}
\frac{\partial E^{\prime}}{\partial t} & =\frac{d}{d t} f_{F_{1}}-f_{2}(x(t)) \mathrm{dvol}(t) \\
& =-f \mathrm{~d} f_{2}\left(x^{\prime}(t)\right)-f f_{2}(x(t)) H_{x^{\prime}(t)}+f f_{2}(x(t)) f H_{x^{\prime}(t)},
\end{aligned}
$$

where $H_{x^{\prime}(t)}$ is the mean curvature of $F_{1, \gamma(t)}$ along horizontal vector $x^{\prime}(t)$.

By (2.2.2), the vector $f_{F_{1, p}} \mathrm{~d} f_{2}\left(x^{\prime}(0)\right)$ has norm in

$$
\left[e^{-\varkappa_{1}\left(\epsilon \mid L_{0}, \delta_{0}, n\right)} L_{0}^{-1}, e^{\varkappa_{1}\left(\epsilon \mid L_{0}, \delta_{0}, n\right)} L_{0}\right] .
$$

At the same time, by (1.3.1) and (3.1.3),

$$
\left|f_{2}(x(t)) H_{x^{\prime}(t)}\right| \leq(m-n) L_{0} e^{\epsilon} \delta \epsilon .
$$

So is the last term in (4.1.5). 
Then for sufficient small $\epsilon,\left.\frac{\partial E^{\prime}}{\partial t}\right|_{t=0}$ has norm

$$
\left|\frac{\partial E^{\prime}}{\partial t}\right|_{t=0} \in\left[e^{-\varkappa_{2}(\epsilon)} L_{0}^{-1}, e^{\varkappa_{2}(e)} L_{0}\right] .
$$

On the other hand, $\partial_{y} E^{\prime}$ equals to the Hessian of $E_{p}(y)$, which by standard Hessian comparison, satisfies

$$
\cos \left(L_{0}^{-1} e^{-\epsilon} \epsilon\right) h_{2} \leq \operatorname{Hess}\left(E_{p}\right) \leq \cosh \left(L_{0} e^{\epsilon} \epsilon\right) h_{2} .
$$

Combining (4.1.4), (4.1.6) and (4.1.7), we conclude that $d \Psi$ has norm

$$
|\mathrm{d} \Psi| \in\left[\left(e^{-\varkappa(\epsilon)} L_{0}\right)^{-1}, e^{\varkappa(\epsilon)} L_{0}\right] .
$$

Now it is easy to see that $\Psi$ is a $e^{\varkappa(\epsilon)} L_{0}$-bi-Lipschitz diffeomorphism and satisfies the requirements in Proposition 4.1. Indeed, by construction $\Psi$ is also an $\left(e^{2 \epsilon} L_{0}, 2 e^{\epsilon} L_{0} \epsilon\right)$-quasi-isometry. It follows from (1.6.1) that the fiber of $\Psi$ has diameter $\leq 2 e^{3 \epsilon} L_{0} \epsilon$. Since (1.1.1) is a local trivialization, the fiber of $\Psi$ must be connected. Hence $\Psi$ is a bi-Lipschitz diffeomorphism.

If the fiber bundles $f_{i}(i=1,2)$ are $G$-equivariant, then by the construction above, it is clear that $\Psi$ is also $G$-equivariant.

Remark 4.2. If in addition, the higher derivatives of $f_{i}$ and the curvature tensor of $Y_{2}$ admit uniform bounds, then so is for $\Psi:\left(Y_{1}, h_{1}\right) \rightarrow\left(Y_{2}, h_{2}\right)$ in Proposition 4.1. In particular, $f_{1}$ and $\Psi^{-1} \circ f_{2}$ would be $\varkappa(\epsilon)$ - $C^{1}$-close.

\section{BundLE ISOMORPHISM $\Phi_{1}$ ON TOTAL SPACES}

In this section we construct a diffeomorphic bundle map

$$
\Phi_{1}:\left(M, Y_{1}, \Psi^{-1} \circ f_{2}\right) \rightarrow\left(M, Y_{1}, f_{1}\right) .
$$

Continue from section 4. Let $\Psi:\left(Y_{1}, h_{1}\right) \rightarrow\left(Y_{2}, h_{2}\right)$ be the bi-Lipschitz diffeomorphism provided by Proposition 4.1. Then the composition $\hat{f}_{2}=\Psi^{-1} \circ f_{2}$ : $\left(M, g_{1}\right) \rightarrow\left(Y_{1}, h_{1}\right)$ is a $\left(\varkappa(\epsilon)+2 \ln L_{0}\right)$-almost Riemannian submersion, which by (4.1.1) satisfies

$$
d\left(f_{1}(x), \hat{f}_{2}(x)\right) \leq 2 L_{0}^{2} \operatorname{diam}_{h_{1}} F_{1, f_{1}(x)} .
$$

Throughout this section, we only use $g_{1}$ and $h_{1}$ and all norms are measured by them.

For any $p \in Y_{1}$ and $x \in F_{2, p}=\hat{f}_{2}^{-1}(p)$, let $p_{x}=f_{1}(x)$. Up to a blowup rescaling, we assume that inj. $\operatorname{rad}_{h_{1}}(p)=1$. Then by (5.1.1), (3.1.2) and (1.5.1),

$$
d\left(p_{x}, p\right) \leq 2 L_{0}^{2} \epsilon \cdot \text { inj. } \operatorname{rad}_{h_{1}}\left(p_{x}\right) \leq \frac{2 L_{0}^{2} \epsilon}{1-2 L_{0}^{2} \epsilon} \text { inj. } \operatorname{rad}_{h_{1}}(p) .
$$

Thus, the minimal geodesic $\gamma_{x}:[0,1] \rightarrow Y_{1}$ from $p_{x}=\gamma_{x}(0)$ to $p=\gamma_{x}(1)$ in $\left(Y_{1}, h_{1}\right)$ is unique and depends smoothly on $x$. Let $\tilde{\gamma}_{x}:[0,1] \rightarrow\left(M, g_{1}\right)$ be the unique $f_{1}$-horizontal lifting of $\gamma_{x}$ at $x$, then we define $\Phi_{1}(x)=\tilde{\gamma}_{x}(1)$.

Clearly, $\Phi_{1}$ depends smoothly on $x$ and lies in $F_{1, p}$, and thus it is a bundle map from $\left(M, Y_{1}, \hat{f}_{2}\right)$ to $\left(M, Y_{1}, f_{1}\right)$, i.e., $f_{1} \circ \Phi_{1}=\hat{f}_{2}$. 
Notice that if $\mathrm{d} \Phi_{1}$ is non-degenerated at every point $x \in M$, then $\Phi_{1}$ is a covering map homotopic to the identity, and hence a diffeomorphic bundle isomorphism. Indeed, a smooth homotopy $H$ is naturally defined by

$$
H:[0,1] \times M \rightarrow M, \quad H(t, x)=\tilde{\gamma}_{x}(t),
$$

such that $H(0, \cdot)=\operatorname{Id}_{M}$, and $H(1, \cdot)=\Phi_{1}$.

To show $d \Phi_{1}$ is isomorphic, we have the following observation.

Lemma 5.2. $d \Phi_{1}$ is non-degenerate if and only if $d \varphi_{2}(v) \neq 0$, for any $f_{2}$-vertical vector $v$.

Proof. Let $0<r<$ inj. $\operatorname{rad}_{h_{1}}(p)=1$, and let

$$
\varphi: f_{1}^{-1}\left(B_{r}(p)\right) \rightarrow B_{r}(p) \times F_{1, p}, \quad \varphi=\left(f_{1}(x), \varphi_{2}(x)\right)
$$

be a local trivialization of $f_{1}$ centered at $F_{1, p}$ defined in (1.1.1), where $\varphi_{2}$ is its 2nd factor as in (1.1.2). Then by definition,

$$
\Phi_{1}(x)=\tilde{\gamma}_{x}(1)=\varphi_{2}(x), \quad \text { for any } x \in F_{2, p} \cap f_{1}^{-1}\left(B_{r}(p)\right) .
$$

It follows that

$$
\mathrm{d} \Phi_{1}(v)=\mathrm{d} \varphi_{2}(v), \quad \text { for any } f_{2} \text {-vertical vector } v \in \mathcal{V}_{f_{2}}(x) .
$$

Furthermore, by definition

$$
\mathrm{d} \hat{f}_{2}=\mathrm{d} f_{1} \circ \mathrm{d} \Phi_{1},
$$

which implies that the kernel of $\mathrm{d} \Phi_{1}$ is contained in the $f_{2}$-vertical distribution $\mathcal{V}_{f_{2}}$,

$$
\operatorname{ker} \mathrm{d} \Phi_{1} \subset \mathcal{V}_{f_{2}}(x) \text {. }
$$

By (5.2.2) and (5.2.4) we conclude Lemma 5.2.

Proposition 5.3. There is $\epsilon_{0}=\epsilon_{0}\left(L_{0}, \delta_{0}, n\right)>0$ such that if $0 \leq \epsilon \leq \epsilon_{0}$, then $\Phi_{1}:\left(M, g_{1}\right) \rightarrow\left(M, g_{1}\right)$ is a diffeomorphism satisfying $f_{1} \circ \Phi_{1}=\hat{f}_{2}$, and the restriction of $\Phi_{1}$ on every fiber is $e^{\varkappa(\epsilon)}$-bi-Lipschitz.

Proof. Continue from the above discussion. Let $\varphi=\left(f_{1}, \varphi_{2}\right)$ be the local trivialization of $f_{1}$ be defined as (1.1.2). By Lemma 5.2, it suffices to show that for any $g_{1}$-unit vector $v \in \mathcal{V}_{f_{2}}(x), \mathrm{d} \Phi_{1}(v)=\mathrm{d} \varphi_{2}(v) \neq 0$.

Let $v=v^{\perp}+v^{\top}$ be its orthogonal decomposition such that $v^{\perp} \in \mathcal{H}_{f_{1}}(x)$ and $v^{\top} \in \mathcal{V}_{f_{1}}(x)$. By (2.2.1) in Proposition 2.2,

$$
\left|v^{\perp}\right| \leq \varkappa(\epsilon), \quad\left|v^{\top}\right| \geq \sqrt{1-\varkappa^{2}(\epsilon)}
$$

where $\varkappa(\epsilon)=\varkappa\left(\epsilon \mid L_{0}, \delta_{0}, m\right)$ and the norm hereafter is measured in $g_{1}$.

Since $\left|\sec \left(Y_{1}, h_{1}\right)\right| \leq 1, \mathrm{~d} \varphi_{2}$ can be explicitly estimated by variation of horizontal curves. Let $\gamma:[0,1] \rightarrow\left(Y_{1}, h_{1}\right)$ be the minimal geodesic from $p_{x}=f_{1}(x)$ to $p=\hat{f}_{2}(x)$. By (1.2.1)-(1.2.2),

$$
\begin{aligned}
& \left|\mathrm{d} \varphi_{2}\left(v^{\top}\right)\right| \geq e^{-e^{\epsilon} \cdot\left|I I_{F_{1, \gamma}}\right| \cdot d\left(p_{x}, p\right)} \cdot\left|v^{\top}\right|, \\
& \left|\mathrm{d} \varphi_{2}\left(v^{\perp}\right)\right| \leq C e^{3 \epsilon+e^{\epsilon}\left|I I_{1, \gamma}\right| \cdot d\left(p_{x}, p\right)} \cdot\left|A_{1, \gamma}\right| \cdot d\left(p_{x}, p\right) \cdot\left|v^{\perp}\right|,
\end{aligned}
$$


where $\left|I I_{1, \gamma}\right|=\max _{t}\left|I I_{f_{1}^{-1}(\gamma(t))}\right|$ and $\left|A_{1, \gamma}\right|=\max _{t}\left|A_{f_{1}^{-1}(\gamma(t))}\right|$, which by (1.4.2), is bounded by $2 e^{3 \epsilon} \delta_{0}$.

Since $d\left(p_{x}, p\right) \leq \frac{2 L_{0}^{2} \epsilon}{1-2 L_{0}^{2} \epsilon}$, combing (5.3.1)-(5.3.2) we derive

$$
\begin{aligned}
\left|\mathrm{d} \Phi_{1}(v)\right| & \geq\left|\mathrm{d} \varphi_{2}\left(v^{\top}\right)\right|-\left|\mathrm{d} \varphi_{2}\left(v^{\perp}\right)\right| \\
& \geq e^{-\varkappa(\epsilon)} \sqrt{1-\varkappa(\epsilon)}-\varkappa(\epsilon) .
\end{aligned}
$$

Clearly, $\left|\mathrm{d} \Phi_{1}\right|$ admits a similar upper bound.

We make several remarks on Proposition 5.3 in order.

Remark 5.4. For any point $x \in M$, by Proposition 4.1 , inj. $\operatorname{rad}_{h_{1}}\left(f_{1}(x)\right)$ is also uniformly proportional to inj. $\operatorname{rad}_{h_{1}}\left(f_{2}(x)\right)$. By Proposition 5.3, $\operatorname{diam}_{g_{1}} F_{1, f_{1}(x)}$ and $\operatorname{diam}_{g_{2}} F_{2, f_{2}(x)}$ are $e^{\varkappa(\epsilon)} L_{0}$-proportional to each other.

Remark 5.5. The method in this section can be applied as a replacement of [7, Proposition A.2.2] in the construction of a global nilpotent Killing structure in [7], where an isotopy from $\operatorname{Id}_{M}$ to $\Phi_{1}$ is required.

Indeed, in the above case $f_{1}$ and $\hat{f}_{2}$ are $C^{1}$-close (see Remark 4.2). Hence, for any $t \in[0,1]$, the map $H_{t}: M \rightarrow Y_{1}, H_{t}(x)=\gamma_{x}(t)$ is also an almost Riemannian submersion, where $\gamma_{x}:[0,1] \rightarrow Y_{1}$ is the unique minimal geodesic from $f_{1}(x)$ to $\hat{f}_{2}(x)$. By the fact again that everything involved is $C^{1}$-close, it is easy to see that the estimate (5.3.1)-(5.3.2) also works for $\Phi_{t}(x)=\tilde{\gamma}_{x}(t)$. Hence the homotopy $H:[0,1] \times M \rightarrow M, H(t, x)=\Phi_{t}(x)$ is an isotopy from $\operatorname{Id}_{M}$ to $\Phi_{1}$.

Therefore, Proposition 5.3 generalizes Proposition A2.2 in [7]. One benefit of our approach is, the normal injectivity radius of fibers are not required to admit a uniform lower bound.

Remark 5.6. Behind the proof of Proposition 4.1 is the fact that $\psi_{p, x}$ defined in (2.3.1) is a diffeomorphism onto its image (though it is not explicitly used). This fact can be verified as follows.

Let $\varphi=\left(f_{1}, \varphi_{2}\right)$ be the local trivialization of $f_{1}$ given by (1.1.1). By the same argument in proving Proposition 5.3, $\mathcal{V}_{f_{2}}$ is transversal to the kernel of $\mathrm{d} \varphi_{2}$ in $f_{1}^{-1}\left(B_{r}(p)\right)$ for any $0<r<\min \left\{1\right.$, inj. $\left.\operatorname{rad}_{h_{1}}(p)\right\}$ and sufficient small $\epsilon$. By Lemma 5.2 , the map $\psi_{p, x}$ is a local diffeomorphism.

We claim that any $f_{2}$-fiber in $f_{1}^{-1}\left(B_{r}(p)\right)$ intersects with $S_{x}=\varphi_{2}^{-1}(x)$ at most once. Hence $\psi_{p, x}$ is a diffeomorphism onto its image.

To verify the claim, let us argue by contradiction. If some $f_{2}$-fiber $F_{2}$ lying in $f_{1}^{-1}\left(B_{r}(p)\right)$ intersects with $S_{x}$ at two points $z_{1}$ and $z_{2}$, then there are two curves $\alpha:[0,1] \rightarrow S_{x}$ and $\beta:[0,1] \rightarrow F_{2}$, both of which are connecting $z_{1}$ and $z_{2}$, such that they are homotopic to each other with fixed endpoints. After passing to the tangent space of $T_{x} M$, the lifts of $S_{x}$ can be viewed as a coordinate plane $\tilde{S}_{x}$. It follows that the lifting $\tilde{\beta}$ has two endpoints in $\tilde{S}_{x}$, and thus there is some $t_{0} \in(0,1)$ such that $\tilde{\beta}^{\prime}\left(t_{0}\right)$ is tangent to $\tilde{S}_{x}$, which contradicts to the fact that $\mathcal{V}_{f_{2}}$ is transversal to $S_{x}$. 


\section{AfFine Bundle Isomorphism ANd Proofs of MAIN THEOREMS}

We first prove Theorem 2.1.

Let $\left(M, Y_{i}, f_{i}\right)$ be two affine bundles equipped with metrics $g_{i}, h_{i}$ on $M$ and $Y_{i}$ respectively, which satisfy the conditions in Theorem 2.1. Then $f_{i}:\left(M, g_{i}\right) \rightarrow$ $\left(Y_{i}, h_{i}\right)$ are Riemannian submersions and $h_{i}$ is the quotient of $g_{i}$ by the canonical nilpotent Killing structure of $f_{i}$ on $\left(M, g_{i}\right)$.

Because $f_{i}$ is a Riemannian submersion, $\left|\nabla^{2} f_{i}\right|$ is bounded by $\max \left\{\left|I I_{f_{i}}\right|,\left|A_{f_{i}}\right|\right\}$, where $\left|I I_{f_{i}}\right|$ is bounded by (2.1.3), and by O'Neil's formula [28] and (2.1.1), $|A|^{2} \leq$ $\frac{8}{3}$. Proposition 2.2 holds for $f_{i}$.

By Proposition 4.1 and Proposition 5.3, there are diffeomorphic bundle isomorphism $\left(\Phi_{1}, \Psi^{-1}\right)$ between $\left(M, Y_{2}, f_{2}\right)$ and $\left(M, Y_{1}, f_{1}\right)$ i.e., $\Psi^{-1} \circ f_{2}=f_{1} \circ \Phi_{1}$. However, $\mathrm{d} \Phi_{1}$ generally does not preserve the affine connection between $f_{i}$-fibers.

In order to improve $\Phi_{1}$ to an affine bundle isomorphism, we first prove that the group actions induced by the affine structures are $C^{1}$-close.

Let $\mathfrak{n}_{1}$ be the canonical nilpotent Killing structure for affine bundle $f_{1}$, and $\mathfrak{n}_{2}$ be the push forward of that for $f_{2}$ by $\Phi_{1}$ on $M$. Let us fix a point $p \in Y_{1}$, and let $r=$ inj. $\operatorname{rad}_{h_{1}}(p) / 2$. Then $U=f_{1}^{-1}\left(B_{r}\left(p ; h_{1}\right)\right)$ is $\mathfrak{n}_{i}$-invariant. Let $\tilde{U} \stackrel{\pi}{\rightarrow} U$ be the universal cover, $\tilde{f}_{1}=f_{1} \circ \pi$ and $\tilde{f}_{2}=f_{2} \circ \Phi_{1}^{-1} \circ \pi$. Then the pullback $\tilde{n}_{i}$ on $\tilde{U}$ generates two free actions $\rho_{i}$ of simply connected nilpotent Lie groups $N_{i}$, which are left translations on $\tilde{f}_{i}$-fibers. Let $\Lambda$ be the fundamental group of $f_{1}^{-1}(p)=$ $\left(\Psi^{-1} \circ f_{2} \circ \Phi_{1}^{-1}\right)^{-1}(p)$. By Malcev's rigidity theorem (see [34]), $N_{1}$ and $N_{2}$ can be identified to a same group $N$ by the natural isomorphism between their lattice $\Lambda \cap$ $N_{i}$. Moreover, the two actions $\rho_{1}$ and $\rho_{2}$ of $N$ coincide on $\Lambda$.

Lemma 6.1. The two actions $\rho_{i}(i=1,2)$ generated by the pullback $\tilde{\mathfrak{n}}_{i}$ on $\tilde{U}$ are $\varkappa\left(\epsilon \mid L_{0}, \delta_{0}, n\right)-C^{1}$-close.

Proof. Let $\tilde{g}_{1}$ (resp. $\tilde{g}_{2}$ ) be the pullback metric of $g_{1}$ (resp. $\left.\left(\Phi_{1}^{-1}\right)^{*} g_{2}\right)$ on $\tilde{U}$. The action $\rho_{i}$ is isometric with respect to $\tilde{g}_{i}$. Since $I I_{\tilde{f}_{i}}$ with respect to $\tilde{g}_{i}$ is under control, by [3, Proposition 4.6.3] (or [7, Lemma 7.13]),

$$
\text { inj. } \operatorname{rad}_{\pi^{*} h_{i}}(\tilde{x}) \geq \min \left\{r / 2, i_{0}\left(\delta_{0}, n\right)\right\}>0,
$$

for any point $\tilde{x}$ with $d(\tilde{x}, \partial \tilde{U})>r / 2$.

Let $\hat{\varepsilon}=\min \left\{1\right.$,inj. $\left.\operatorname{rad}_{h_{1}}(p)\right\}$. Let us rescale $\tilde{g}_{i}$ by $\hat{\varepsilon}^{-1}$ and let $\epsilon \rightarrow 0$. By passing to a subsequence, we can assume that the equivariant convergence

$$
\left(\tilde{U}, \hat{\varepsilon}^{-1} \tilde{g}_{i}, x, \rho_{i}(N)\right) \stackrel{C^{1, \alpha}}{\longrightarrow}\left(\tilde{U}, \tilde{g}_{i, \infty}, x, \rho_{i, \infty}(N)\right) .
$$

Because the diameter of both $f_{1}$-fibers and $f_{2}$-fibers goes to 0 , the action of $\Lambda$ becomes more and more dense such that $\rho_{i, \infty}(N)$ is also the limit action of $\Lambda$. Thus the two limit actions coincide. This implies that the actions of $\tilde{\mathfrak{n}}_{i}$ are $C^{1}$-close, which are invariant under rescaling by $\hat{\varepsilon}$.

By the $C^{1}$-closeness of $\rho_{i}$, it follows from the argument in [7, section 7] that $\Phi_{1}$ can be modified to an affine bundle isomorphism. In the following we give a proof for completeness. 
Proposition 6.2. There is an G-equivariant diffeomorphism $\Phi_{2}: M \rightarrow M$ such that $\left(\Phi_{2}\right)_{*} \mathfrak{n}_{2}=\mathfrak{n}_{1}$.

Proof. We will follow the argument in [7, section 7] to derive $\Phi_{2}$. Let $\tilde{U}_{1}=$ $\tilde{f}_{1}^{-1}\left(B_{r / 2}(p)\right) \subset \tilde{U}$

We first prove that there is a $N$-equivariant diffeomorphism $\tilde{\Phi}_{2}: \tilde{U}_{1} \rightarrow \tilde{U}_{1}$. Continue from Lemma 6.1. Let $\rho_{i}$ be the action of $N$ on $\tilde{U}$. For any $\lambda \in \Lambda$, we have $\rho_{1}(\lambda)=\rho_{2}(\lambda)$, and for any $[h] \in \Lambda \backslash N, \rho_{1}\left([h]^{-1}\right) \circ \rho_{2}([h])$ is well defined and $\varkappa(\epsilon) C^{1}$-close to the identity. For any $x \in \tilde{U}_{1}$, let $\tilde{\Phi}_{2}(x)$ be the center of mass of $h \mapsto \rho_{1}\left([h]^{-1}\right) \circ \rho_{2}([h])$, i.e., the critical value of

$$
y \rightarrow \int_{\Lambda \backslash N} d\left(y, \rho_{1}\left(h^{-1}\right) \circ \rho_{2}(h) x\right) d h
$$

By [19], $\tilde{\Phi}_{2}: \tilde{U}_{1} \rightarrow \tilde{U}_{1}$ is well-defined diffeomorphism such that

$$
\tilde{\Phi}_{2} \circ \rho_{2}(h)(x)=\rho_{1}(h) \tilde{\Phi}_{2}(x), \quad \text { for any } h \in N .
$$

Secondly, by the construction, the quotient $\Phi_{2}: f_{1}^{-1}\left(B_{r / 2}(p)\right) \rightarrow f_{1}^{-1}\left(B_{r / 2}(p)\right)$ is $G$-equivariant and affine-equivariant. Moreover, for any $x \in B_{r / 2}(p)$, the definition of $\Phi_{2}(x)$ does not depends on the choice of $p$. Thus $\Phi_{2}$ can be extended to a globally defined $G$-equivariant diffeomorphism such that $\left(\Phi_{2}\right)_{*} \mathfrak{n}_{2}=\mathfrak{n}_{1}$.

\section{Proof of Theorem 2.1.}

Let $\Psi: Y_{1} \rightarrow Y_{2}, \Phi_{1}: M \rightarrow M$ and $\Phi_{2}: M \rightarrow M$ to be diffeomorphism given by Proposition 4.1, Proposition 5.3 and Proposition 6.2 respectively. Let $\Phi=\left(\Phi_{2} \circ \Phi_{1}\right)^{-1}$, then $\Psi \circ f_{1}=f_{2} \circ \Phi$. Because $\Phi$ preserves the nilpotent Killing structures of $f_{i}$, it is an affine bundle isomorphism. By construction, $\Phi$ is $G$-equivariant, if in addition, $f_{i}$ are $G$-equivariant.

\section{Proof of Theorem 0.1.}

Let $\mathfrak{n}_{i}$ be a nilpotent Killing structure on $M$ associated to $g_{i}(i=1,2)$. By Theorem 1.11, without loss of generality we assume that $g_{i}$ is $\mathfrak{n}_{i}$-invariant and $\left|\nabla^{j} R\right| \leq A_{j}(n)(j=0,1, \ldots)$. By O'Neill formula, the orthonormal frame bundle $\left(F M, \tilde{g}_{i}\right)$, with a canonical metric induced by $g_{i}$, still admit a uniform two-sided sectional curvature bound.

Since the differential action of $\mathfrak{n}_{i}$ on $F M$ is free and $\mathfrak{n}_{i}$ is pure, the quotient of $F M$ by the lifting nilpotent Killing structure $\tilde{\mathfrak{n}}_{i}$ is still a Riemannian manifold $Y_{i}$. Moreover, since $\mathfrak{n}_{i}$ is pure and points all collapsing directions, the injectivity radius of $Y_{i}$ admits a uniform lower bound $i_{0}(n)$ (see [13], [7]).

Thus, $\tilde{\mathfrak{n}}_{i}$ corresponds to an $O(n)$-equivariant affine bundle $\tilde{f}_{i}:\left(F M, \tilde{g}_{i}\right) \rightarrow Y_{i}$ satisfying (2.1.1), (2.1.2), and (2.1.3).

If the dimension of $Y_{i}$ is the same, then by Theorem 2.1, $Y_{1}$ and $Y_{2}$ are diffeomorphic and $\left(F M, Y_{i}, \tilde{f}_{i}\right)$ are isomorphic as affine bundles. Consequently, the affine bundle isomorphism $\tilde{\Phi}: F M \rightarrow F M$ descends to a diffeomorphism $\Phi: M \rightarrow$ $M$, such that $\Phi_{*} \mathfrak{n}_{1}=\mathfrak{n}_{2}$, and their infinitesimal action are conjugate by $\Phi$. 
Now let us assume that $n_{1}=\operatorname{dim} Y_{1}<\operatorname{dim} Y_{2}=n_{2}$. Since $g_{1}$ and $g_{2}$ are $L_{0^{-}}$ Lipschitz equivalent, the nilpotent Killing structure for $\tilde{g}_{2}$ can be chosen to be of the same dimension, i.e., there is another affine bundle $\tilde{f}_{2}^{\prime}:\left(F M, \tilde{g}_{2}\right) \rightarrow\left(Y^{\prime}\right)^{n_{1}}$.

By local compatibility of nilpotent structures (see [7, Section 7]), there is an affine bundle $\varphi: Y^{n_{2}} \rightarrow\left(Y^{\prime}\right)^{n_{1}}$ such that $\varphi \circ \tilde{f}_{2}$ is isomorphic to $\tilde{f}_{2}^{\prime}$ as affine bundles. Now by Theorem 2.1, $\tilde{f}_{2}^{\prime}$ is conjugate to $\tilde{f}_{1}$, and thus after descending to $M$, we derive a diffeomorphism $\Phi: M \rightarrow M$ such that $\Phi_{*} \mathfrak{n}_{2} \subset \mathfrak{n}_{1}$ as a subsheaf.

Proof of Theorem 0.3.

It directly follows from Theorem 0.1 .

\section{REFERENCES}

[1] Michael T. Anderson. Hausdorff perturbations of Ricci-flat manifolds and the splitting theorem. Duke Math. J., 68(1):67-82, 101992.

[2] D. Burago, Y. Burago, and S. Ivanov. A Course in Metric Geometry. Graduate Studies in Mathematics, vol. 33. American Mathematical Society, Providence, Rhode Island, 2001.

[3] P. Buser and H. Karcher. Gromov's almost flat manifolds. Astérisque, 81. Société mathématique de France, 1981.

[4] Q.S. Cai and X.C. Rong. Collapsing construction with nilpotent structures. Geometric and Functional Analysis, 18(5):1503-1524, Feb 2009.

[5] J. Cheeger. Comparison and finiteness theorems for Riemannian manifolds. PhD thesis, Princeton University, 1967.

[6] J. Cheeger. Finiteness theorems for Riemannian manifolds. American Journal of Mathematics, 92(1):61-74, 1970.

[7] J. Cheeger, K. Fukaya, and M. Gromov. Nilpotent structures and invariant metrics on collapsed manifolds. Journal of the American Mathematical Society, 5(2):327-372, 1992.

[8] J. Cheeger and M. Gromov. Collapsing Riemannian manifolds while keeping their curvature bounded I. J. Differential Geom., 23:309-346, 1986.

[9] J. Cheeger and M. Gromov. Collapsing Riemannian manifolds while keeping their curvature bounded. II. J. Differential Geom., 32(1):269-298, 1990.

[10] X.Z. Dai, G.F. Wei, and R.G. Ye. Smoothing Riemannian metrics with Ricci curvature bounds. manuscripta mathematica, 90(1):49-61, 1996.

[11] F.Q. Fang and X.C. Rong. Positive pinching, volume and second betti number. Geometric \& Functional Analysis GAFA, 9(4):641-674, 1999.

[12] K. Fukaya. Collapsing Riemannian manifolds to ones with lower dimensions. J. Differential Geom., 25:139-156, 1987.

[13] K. Fukaya. A boundary of the set of the Riemannian manifolds with bounded curvatures and diameters. J. Differential Geom., 28:1-21, 1988.

[14] K. Fukaya. Collapsing Riemannian manifolds to ones with lower dimension II. Journal of The Mathematical Society of Japan, 41(2):333-356, 1989.

[15] K. Fukaya. Metric Riemannian geometry. In Handbook of Differential Geometry, Volume 2, pages 189-313. Elsevier B.V., 2006.

[16] R. E. Green and H. Wu. Lipschitz convergence of Riemannian manifolds. Pacific J. Math., 131:119-141, 1988.

[17] M. Gromov. Almost flat manifolds. J. Differential Geom., 13(2):231-241, 1978.

[18] M. Gromov, J. Lafontaine, and P. Pansu. Structures mètriques pour les variètès riemanniennes. (French) [Metric structures for Riemann manifolds]. Textes Mathlęmatiques [Mathematical Texts], 1. CEDIC/Fernand Nathan, Paris, 1981.

[19] K. Grove and H. Karcher. How to conjugate $C^{1}$-close group actions. Mathematische Zeitschrift, 132(1):11-20, Mar 1973. 
[20] R. S. Hamilton. Three-manifolds with positive Ricci curvature. J. Differential Geom., 17(2):255-306, 1982.

[21] H.Z. Huang, L.L. Kong, X.C Rong, and S.C. Xu. Collapsed manifolds with Ricci bounded below and local covering geometry. in preparation.

[22] H.Z. Huang, L.L. Kong, X.C Rong, and S.C. Xu. Collapsed manifolds with Ricci bounded covering geometry. preprint, May 2018.

[23] V. Kapovitch. Perelman's stability theorem. In Metric and Comparison Geometry, volume XI of Surveys in Differential Geometry, pages 103-136. International Press of Boston, 2007.

[24] Atsushi Kasue. A convergence theorem for Riemannian manifolds and some applications. Nagoya Math. J., 114:21-51, 1989.

[25] X. Li and S.C. Xu. A parametrized compactness theorem under bounded Ricci curvature. Frontiers of Mathematics in China, 13(1):67-85, 2018.

[26] Xiang Li. The Stability of fiber bundles on collapsing manifolds. PhD thesis, Capital Normal University, China, 2011.

[27] Ye Li. Smoothing Riemannian metrics with bounded Ricci curvatures in dimension four. Advances in Mathematics, 223(6):1924 - 1957, 2010.

[28] Barrett O'Neill. The fundamental equations of a submersion. Michigan Math. J., 13(4):459-469, 1966.

[29] H. Ozeki and F. Uchida. Principal circle actions on a product of spheres. Osaka J. Math., 9:379_ 390, 1972.

[30] S. Peters. Convergence of Riemannian manifolds. Compositio Mathematica, 62(1):3-16, 1987.

[31] P. Petersen, G. Wei, and R. Ye. Controlled geometry via smoothing. Commentarii Mathematici Helvetici, 74(3):345-363, Sep 1999.

[32] A. Petrunin, X.C. Rong, and W. Tuschmann. Collapsing vs. positive pinching. Geometric \& Functional Analysis GAFA, 9(4):699-735, 1999.

[33] A. Petrunin and W. Tuschmann. Diffeomorphism finiteness, positive pinching, and second homotopy. Geometric \& Functional Analysis GAFA, 9(4):736-774, 1999.

[34] M. Raghunathan. Discrete subgroup of Lie groups. Springer, 1972.

[35] X.C. Rong. Collapsed manifolds with bounded sectional curvature and applications. In J. Cheeger and K. Grove, editors, Surveys in Differential Geometry, Vol. XI, volume XI, pages 1-23. International Press, Somerville, MA, 2007.

[36] X.C. Rong and S.C. Xu. Stability of $e^{\epsilon}$-lipschitz and co-lipschitz maps in gromov-hausdorff topology. Advances in Mathematics, 231(2):774-797, 2012.

[37] Ernst A. Ruh. Almost flat manifolds. J. Differential Geom., 17(1):1-14, 1982.

[38] M. Y. Wang and W. Ziller. Einstein metrics on principal torus bundles. J. Differential Geom., 31(1):215-248, 1990.

[39] S.C. Xu. Local estimate on convexity radius and decay of injectivity radius in a riemannian manifold. Communications in Contemporary Mathematics, July 2017.

School of Mathematical Sciences, Capital Normal Universiy, BeiJing China

E-mail address: jiangzuohai08@163.com

School of Mathematical Sciences, Capital Normal Universiy, BeiJing China

E-mail address: shichengxu@gmail.com 